\title{
Methylene Blue for Vasoplegic Syndrome
}

\author{
Alexander T. Booth, BA, Patrick D. Melmer, MD, J. Benjamin Tribble, BA, J. Hunter Mehaffey, MD, \\ Curt Tribble, MD \\ Division of Thoracic and Cardiovascular Surgery, Department of Surgery, University of Virginia, Charlottesville, Virginia, USA
}

\section{ABSTRACT}

Vasoplegic syndrome is a form of vasodilatory shock that occurs frequently in patients who undergo cardiac surgery requiring cardiopulmonary bypass (CBP). Treatment often demands high doses of vasopressors over sustained periods for hypotension that can be refractory to standard vasoactive medications. Furthermore, the development of vasoplegia greatly contributes to morbidity and mortality following cardiac surgery. Methylene blue $(\mathrm{MB})$ has become a popular therapy for cardiac vasoplegia despite a paucity of prospective data to direct its use. Therefore, the aim of this study was to review available data regarding mechanisms, dosing strategies, and side effects of $M B$, with a focus on its applications for vasoplegia in cardiac surgery.

\section{INTRODUCTION}

Methylene blue $(\mathrm{MB})$ is a tricyclic phenothiazine that inhibits guanylate cyclase (GC). Historically known as a dye, $\mathrm{MB}$ has been applied in multiple areas of medicine including management of methemoglobinemia, septic shock and lymphatic mapping for sentinel lymph node biopsy. There is a growing body of evidence to suggest $\mathrm{MB}$ is an effective therapy for vasoplegic syndrome associated with cardiac surgery and cardiopulmonary bypass.

Although there is no consensus definition for vasoplegic syndrome, it occurs with significant frequency in the setting of cardiac surgery. The syndrome is characterized by a sepsis-like hemodynamic state with loss of arterial tone and profound hypotension than can be refractory to vasopressors. Proposed criteria include mean arterial pressure $<50 \mathrm{mmHg}$, systemic vascular resistance (SVR) $<800$ dynes-sec- $\mathrm{cm}^{5}$, cardiac index $>2.5 \mathrm{~L} / \mathrm{min} / \mathrm{m} 2$, right atrial pressure $<5 \mathrm{mmHg}$, and left atrial pressure $<10 \mathrm{mmHg}$ despite vasopressor infusion [Riha 2011], [Patarroyo 2012]. Incidence reports vary, but one recent series identified 638 consecutive cardiac surgery patients and found that $56(8.8 \%)$ experienced vasoplegia [Levin 2004]. The authors reported decreased morbidity and mortality for vasoplegic patients who were treated with MB. However, another retrospective analysis of 57 patients who received $\mathrm{MB}$ after cardiac surgery and CBP recommended it

Received March 27, 2017; received in revised form fuly 31, 2017; accepted August 7, 2017.

Correspondence: Curt Tribble, MD, Department of Surgery, University of Virginia School of Medicine, PO Box 800300, Charlottesville, VA 22908; (e-mail: CGT2E@bscmail.mcc.virginia.edu). for "rescue use" only as it was associated with worse outcomes after propensity-scored matching [Weiner 2013].

In this review, the authors summarize what is currently known about the mechanisms, dosing strategies, and potential side effects of $\mathrm{MB}$, with a focus on its applications for vasoplegia associated with cardiac surgery.

\section{THE VASOPLEGIC SYNDROME}

Although vasoplegic syndrome has been reported in offpump cardiac surgery [Raja 2004], it is primarily associated with the systemic inflammatory response that occurs from bypass itself [Cremer 1996]. In a series of patients undergoing coronary artery bypass grafting (CABG), patients managed on CPB had increased levels of TNF-a, IL-6, and IL-8, as well as increased complement levels compared with off-pump controls [Strüber 1999]. This inflammatory response results in transient vascular dysfunction with vasodilation, hypotension, and resistance to vasopressors that can cause profound organ hypoperfusion.

One of the first descriptions of vasoplegic syndrome following CPB during cardiac surgery was published in 1994 [Gomes 1994]. Gomes et al described a series of patients who experienced hypotension with normal or elevated CO, decreased filling pressures, and low SVR in the early postoperative period. Fluid resuscitation alone was insufficient in restoring normal hemodynamics, and patients often required high doses of norepinephrine for prolonged periods of time, up to several days in some. Morbidity and mortality in this group was very high.

A few years later, in the late 1990s and early 2000s, multiple investigators described methylene blue as an effective therapy for vasoplegia in such patients. Yiu and colleagues reported a patient with vasoplegia refractory to high doses of norepinephrine following CABG. Intravenous administration of $\mathrm{MB}$ dosed at $2 \mathrm{mg} / \mathrm{kg}$ over 20 minutes decreased the dose of norepinephrine required to maintain a systolic blood pressure of $90 \mathrm{mmHg}$ after 45 minutes [Yiu 1999]. Evora authored several papers that also promoted the use of $\mathrm{MB}$ for patients during and after $\mathrm{CPB}$ and in anaphylactic shock [Andrade 1996; Evora 1997; Evora 2000]. Though the mechanism was not fully elucidated, these preliminary studies demonstrated that there appeared to be a "window of opportunity" for efficacious use of MB [Evora 2014](Table).

\section{MECHANISM}

Excess nitric oxide (NO) production is central to the pathophysiology of both septic shock and vasoplegic syndrome. 
Summary of Studies Investigating Novel Treatments for Vasoplegic Syndrome Related to Cardiac Surgery

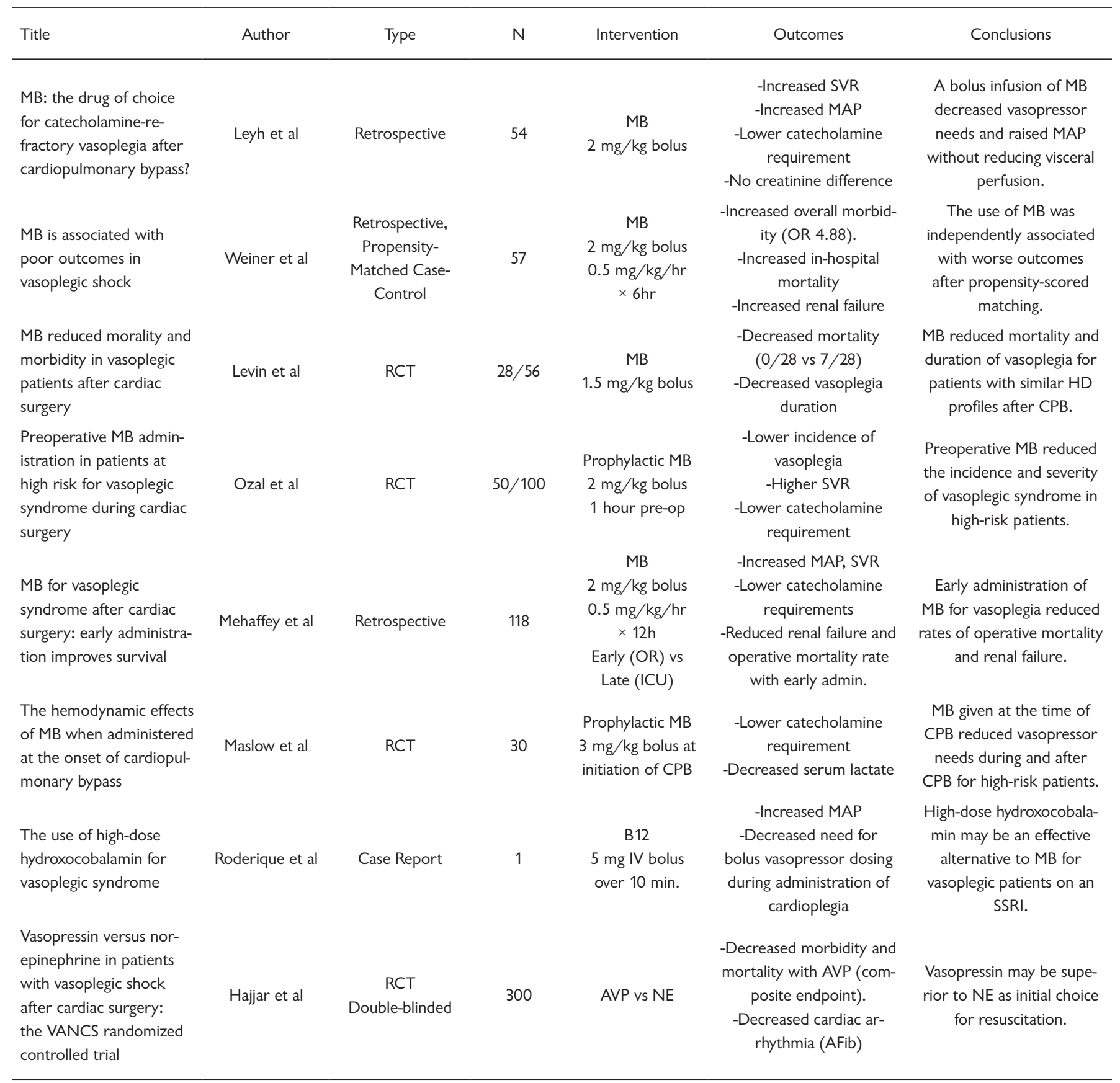

MB indicates methylene blue; RCT, randomized controlled trial; AVP, arginine vasopressin; NE, norepinephrine; SVR, systemic vascular resistance; MAP, mean arterial pressure; CPB, cardiopulmonary bypass; HD, hemodynamic; SSRI, selective serotonin reuptake inhibitor.

Increased NO synthase activity in an inflammatory state leads to release of $\mathrm{NO}$ by endothelial cells. NO activates soluble guanylyl cyclase (sGC), resulting in higher cyclicguanosine monophosphate (c-GMP) concentrations and subsequent vessel relaxation. Methylene blue is an inhibitor of soluble GC and thus attenuates vasodilation mediated by the NO pathway in the setting of shock (Figure). A prospective clinical trial of intravenous $\mathrm{MB}$ administration in septic shock demonstrated progressive increases in mean arterial pressure associated with an improvement in cardiac function [Preiser 1995].

This proposed mechanism has proven controversial, however. Myles and colleagues measured plasma and urine nitrate levels as markers for NO production in patients who had 


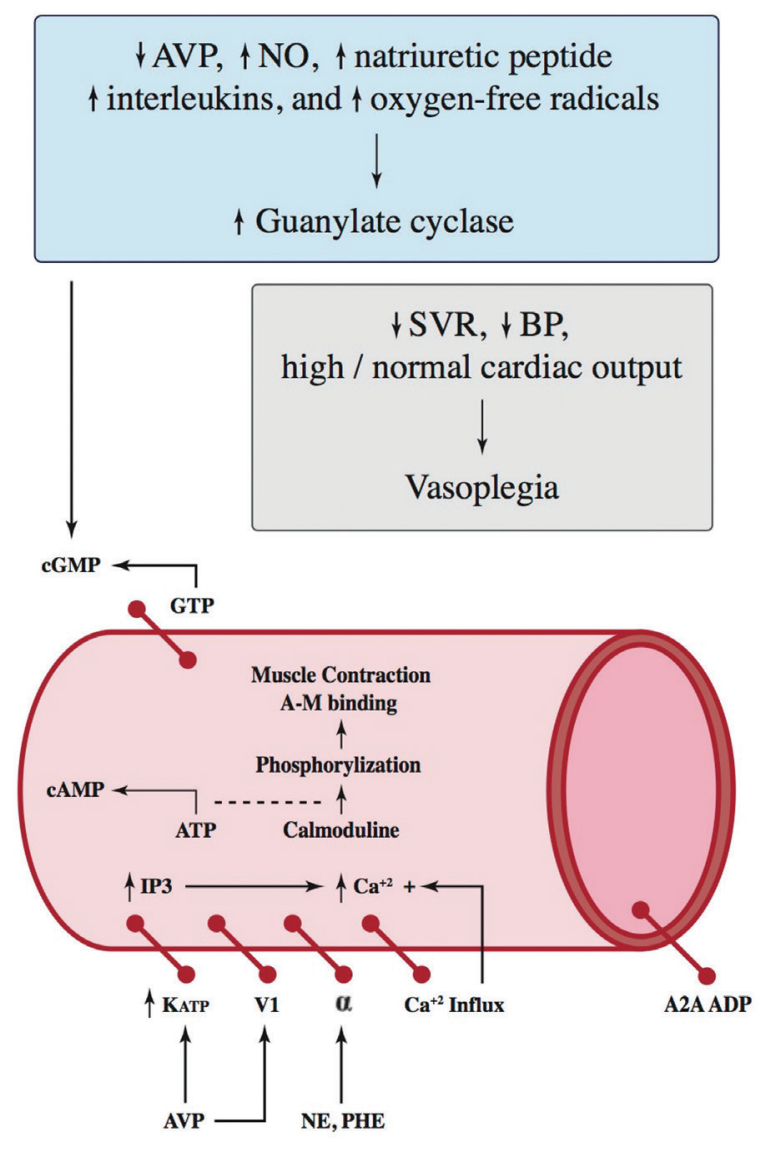

Pathophysiology of vasoplegic syndrome.

undergone CPB. They found no significant difference in nitrate levels between hypotensive cases and normotensive controls [Myles 1997]. Other possible mechanisms for the ability of MB to reverse vessel relaxation include a non-nitric c-GMP pathway or another type of receptor, which may explain why increased levels of $\mathrm{NO}$ were not found in these studies.

\section{ADMINISTRATION TIMING \& DOSING}

Recommended dosing strategies for MB vary, but many authors describe a dose of $2 \mathrm{mg} / \mathrm{kg}$ delivered intravenously over 30 minutes. The first report to describe MB following heart transplantation used this dosing regimen with good results. The patient, a 55-year-old man with congestive heart failure, who underwent successful orthotopic cardiac transplantation, experienced vasoplegia postoperatively in the cardiac intensive care unit and required norepinephrine up to $1 \mu \mathrm{g} / \mathrm{kg} / \mathrm{min}$ in order to maintain a systolic blood pressure of $100 \mathrm{mmHg}$. $\mathrm{MB}$ administration at $2 \mathrm{mg} / \mathrm{kg}$ for 30 minutes resulted in an immediate increase in blood pressure. The norepinephrine infusion was also weaned to $0.22 \mu \mathrm{g} / \mathrm{kg} / \mathrm{min}$ in 60 minutes and discontinued at 6 hours [Kofidis 2001]. In another series, 54 patients with norepinephrine-refractory vasoplegia during or after CPB were treated with MB $2 \mathrm{mg} / \mathrm{kg}$ over 20 minutes. 51 patients (94\%) experienced a clinically significant increase in SVR and associated decrease in norepinephrine requirements after 1 hour of treatment. No side effects were noted [Leyh 2003]. The protocol used at this academic institution for similar circumstances involves $2 \mathrm{mg} / \mathrm{kg}$ bolus of intravenous $\mathrm{MB}$, followed by a 12 -hour infusion at $0.5 \mathrm{mg} / \mathrm{kg} / \mathrm{hr}$. A recently published study demonstrated improved survival for vasoplegia when $\mathrm{MB}$ was administered in the early (operating room) versus late (intensive care) postoperative setting $(10.4 \%$ versus 28.6\%, $P=.018$ ) [Mehaffey 2017].

Most uses of $\mathrm{MB}$ have been described for patients recovering in a postoperative cardiac ICU with vasoplegia refractory to standard resuscitative efforts, yet there has also been some success with prophylactic dosing in the pre- and perioperative periods. Ozal et al performed the first of only two prospective studies describing $\mathrm{MB}$ administration in cardiac surgery [Ozal 2005]. They selected patients at high risk for developing vasoplegia based on preoperative use of angiotensin-converting enzyme inhibitors, calcium channel blockers, or heparin. None of the patients who received MB preoperatively developed vasoplegia compared with $26 \%$ in the untreated control group. They reported a significant reduction in morbidity and mortality within the treated group as well as reduced ICU and hospital lengths of stay. A subsequent prospective trial redemonstrated these findings while showing that serum lactate levels were lower in patients given $\mathrm{MB}$, suggesting enhanced tissue perfusion [Maslow 2006]. Taken together, these studies suggest that better identification of risk factors for vasoplegia with prophylactic $\mathrm{MB}$ administration may improve outcomes for high-risk groups, such as those undergoing LVAD placement or patients taking heparin.

The available data concerning risk factors for vasoplegia including type of surgery, comorbidities, medications, and duration of CPB have been reviewed elsewhere [Omar 2015]. Of note, individual study results are highly dependent on each author's definition of vasoplegia. Thus, reported incidence and associated risk factors vary with the population of interest (eg, heart failure versus CAD) as well as how each study identifies patients with vasoplegia [Hajjar 2017]. Standardized criteria for defining this syndrome are needed to improve comparison between studies and better determine high-risk groups that may benefit from prophylactic MB.

\section{TOXICITY, SIDE EFFECTS \& ALTERNATIVES}

The most common side effects of $\mathrm{MB}$ administration are self-limited blue-green cutaneous staining and urine discoloration. It can also cause hemolytic anemia in patients with G6PD deficiency and decrease oximetry measurements without changing $\mathrm{PaO} 2$. Most importantly, precipitation of the serotonin syndrome must be considered prior to use of $\mathrm{MB}$ in combination with drugs that inhibit serotonin reuptake. Though rare, such cases have been described [Grubb 2012; Hanna 2014; Hencken 2016]. Following MB administration for refractory hypotension during heart transplantation, a 60 -year-old male experienced delayed recovery from anesthesia and was found to have ocular clonus, mydriasis, horizontal nystagmus, hypertonicity, temperature to 40 degrees Celsius 
and hypoxia. As is the case for many LVAD patients awaiting transplantation, his comorbidities included depression, for which he was taking $20 \mathrm{mg}$ escitalopram qd and $100 \mathrm{mg}$ trazadone qhs. As there is no specific treatment for serotonin syndrome, the patient was re-intubated and sedated with benzodiazepines to allow for clearance of the MB. After 48 hours, the patient was weaned from the ventilator, extubated, and ultimately recovered uneventfully [Grubb 2012]. The authors note that within three years at their institution, 18 of 47 patients $(38 \%)$ who underwent heart transplantation after LVAD were taking an SSRI at the time of their operation. A similar case described a patient who developed serotonin syndrome following MB administration and who subsequently suffered a cardiac arrest. Following successful cardiopulmonary resuscitation, the patient ultimately recovered without permanent neurological deficits [Hanna 2014]. Surgeons and intensive care specialists should be aware of these drug interactions and avoid $\mathrm{MB}$ in the setting of serotonin reuptake inhibition [Gillman 2011].

Given the prevalence of comorbid depression and SSRI use in the cardiac surgery population, alternatives to $\mathrm{MB}$ for vasoplegia are being pursued. High-dose hydroxocobalamin (B12) has been in use for decades as a treatment for cyanide poisoning. One side effect of a $5 \mathrm{~g}$ dose (Cyanokit) is a significant, sustained increase in blood pressure as a result of direct nitric oxide binding and inhibition of NO synthase and guanylate cyclase. In one case of hypotension on CPB during cardiac surgery, vasoplegia was treated with $5 \mathrm{mg}$ of IV B12 over 10 minutes with a rapid, sustained blood pressure response and reduction in vasopressor requirements [Roderique 2014]. Unlike MB, it has no effect on serotonin metabolism and has been suggested as an alternative for patients on SSRIs undergoing cardiac surgery.

\section{FUTURE DIRECTIONS}

The majority of literature on the use of MB for vasoplegia is retrospective in nature and offers somewhat conflicting data. While there are two prospective studies that have looked at the incidence of vasoplegia in patients at risk, and at the outcomes with the use of MB administration, the literature examining MB's true efficacy and outcomes with longterm follow-up are limited. A prospective clinical trial that randomized patients with vasoplegia to usual care versus usual care plus MB would provide valuable guidance in the use of this drug. The development of such a randomized controlled trial will be challenging due to MB's lack of approved indications by the US Food and Drug Administration. The risk of serotonin syndrome coupled with low production costs relative to other drugs are further obstacles to large-scale studies with this medication.

\section{Conclusion}

Vasoplegia is a form of shock that can occur in patients who have undergone $\mathrm{CPB}$ for cardiac surgery and is associated with significant morbidity and mortality in the postoperative period. MB has been advocated as a useful therapeutic agent known for its ability to curb vascular relaxation in the setting of increased NO and c-GMP expression. The currently suggested regimen is $2 \mathrm{mg} / \mathrm{kg}$ given as a loading dose over 20-30 minutes, with a maintenance rate of $0.5 \mathrm{mg} / \mathrm{kg} /$ $\mathrm{hr}$ and $7 \mathrm{mg} / \mathrm{kg}$ maximum. After initial administration, there appears to be decreased effectiveness from continued use [Manghelli 2015; Lavigne 2010]. Further research into this medication is needed, particularly to study the prophylactic preoperative use for patients deemed at higher-than-average risk for development of vasoplegic syndrome.

\section{REFERENCES}

Andrade JCS, Batista ML, Evora PRB, et al. 1996. Methylene blue administration in the treatment of the vasoplegic syndrome after cardiac surgery. Rev Bras Cir Cardiovasc 11:107-14.

Cremer J, Martin M, Redl H, et al. 1996. Systemic inflammatory response syndrome after cardiac operations. Ann Thorac Surg 61:1714-20.

Evora PR, Ribeiro PJ, Andrade JC. 1997. Methylene blue administration in SIRS after cardiac operations. Ann Thorac Surg 63:1212-13.

Evora PR. 2000. Should MB be the drug of choice to treat vasoplegias caused by cardiopulmonary bypass and anaphylactic shock? J Thorac Cardiovasc Surg 119: 632-4.

Evora PRB, Rodrigues AJ, Celotto AC. 2014. Methylene blue should be relegated to rescue use and not as first-line therapy. J Cardiothorac Vasc Anesth 28: 11-12.

Gillman PK. 2011. CNS toxicity involving methylene blue: the exemplar for understanding and predicting drug interactions that precipitate serotonin toxicity. J Psychopharmacol 25:429-36.

Gomes WJ, Carvalho AC, Palma JH, Gonçalves I Jr, Buffolo E. 1994. Vasoplegic syndrome: a new dilemma. J Thorac Cardiovasc Surg 107:942-3

Grubb KJ, Kennedy JL, Bergin JD, Groves DS, Kern JA. 2012. The role of methylene blue in serotonin syndrome following cardiac transplantation: a case report and review of the literature. J Thorac Cardiovasc Surg 144:e113-6.

Hajjar, Vincent JL, Galas FRBG, et al. 2017. Vasopressin versus norepinephrine in patients with vasoplegic shock after cardiac surgery: the VANCS randomized controlled trial. Anesth 126:85-93.

Hanna ER, Clark JA. 2014. Serotonin syndrome after cardiopulmonary bypass: a case demonstrating the interaction between methylene blue and selective serotonin reuptake inhibitors. A A Case Rep 2:113-4.

Hencken L, To L, Ly N, Morgan JA. 2016. Serotonin syndrome following methylene blue administration for vasoplegic syndrome. J Card Surg 31:208-10.

Kofidis T, Strüber M, Wilhelmi M, et al. 2001. Reversal of severe vasoplegia with single-dose methylene blue after heart transplantation. J Thorac Cardiovasc Surg 122:823-4.

Lavigne D. 2010. Vasopressin and methylene blue: alternate therapies in vasodilatory shock. Semin Cardiothorac Vasc Anesth 14:186-9.

Levin RL, Degrange MA, Bruno GF, et al. 2004. Methylene blue reduces mortality and morbidity in vasoplegic patients after cardiac surgery. Annals Thorac Surg 77: 496-9.

Leyh RG, Kofidis T, Strüber M, et al. 2003. Methylene blue: the drug of choice for catecholamine refractory vasoplegia after cardiopulmonary bypass? J Thorac Cardiovasc Surg 125:1426-31. 
Manghelli J, Brown L, Tadros HB, Munfakh NA. 2015. A reminder of methylene blue's effectiveness in treating vasoplegic syndrome after onpump cardiac surgery. Tex Heart Institute J 42:491-4.

Maslow AD, Stearns G, Butala P, Schwartz CS, Gough J, Singh AK. 2006. The hemodynamic effects of methylene blue when administered at the onset of cardiopulmonary bypass. Anesth Analg 103:2-8.

Mehaffey JH, Johnston LE, Hawkins RB, et al. 2017. Methylene blue for vasoplegic syndrome after cardiac operation: early administration improves survival. Ann Thorac Surg 104:36-41.

Myles PS, Leong CK, Currey J. 1997. Endogenous nitric oxide and low vascular resistance after cardiopulmonary bypass. J Cardiothorac Vasc Anesth 11:571-4.

Omar S, Zedan A, Nugent K. 2015. Cardiac vasoplegia syndrome: pathophysiology, risk factors, and treatment. Am J Med Sci 349:80-8.

Ozal E, Kuralay E, Yildirim V, et al. 2005. Preoperative methylene blue administration in patients at high risk for vasoplegic syndrome during cardiac surgery. Ann Thorac Surg 79:1615-9.

Patarroyo M, Simbaqueba C, Shrestha K, et al. 2012. Pre-operative risk factors and clinical outcomes associated with vasoplegia in recipients of orthotopic heart transplant in the contemporary era. J Heart Lung Transpl 31:282-7.

Preiser JC, Lejeune P, Roman A, et al. 1995. Methylene blue administration in septic shock: a clinical trial. Crit Care Med 23:259-64.

Raja SG, Dreyfus GD. 2004. Vasoplegic syndrome after off-pump coronary artery bypass surgery. Tex Heart Inst J 31:421-4.

Riha H, Augoustides JGT. 2011. Pro: Methylene blue as a rescue therapy for vasoplegia after cardiac surgery. J Cardiothorac Vasc Anesth 25:736-8.

Roderique JD, VanDyck K Holman B, et al. 2014. The use of high-dose hydroxocobalamin for vasoplegic syndrome. Ann Thorac Surg 97:1785-6.

Strüber M, Cremer J, Gohrbandt B, Haverich A. 1999. Humoral cytokine response to coronary artery bypass grafting with and without cardiopulmonary bypass. Ann Thorac Surg 68:1330-5.

Weiner MM, Lin H, Danforth D, Rao S, Hosseinian L, Fischer GW. 2013. Methylene blue is associated with poor outcomes in vasoplegic shock. J Cardiothorac Vasc Anesth 27:1233-8.

Yiu P, Robin J, Pattison CW. 1999. Reversal of refractory hypotension with single-dose methylene blue after coronary bypass surgery. J Thorac Cardiovasc Surg 118:195-6. 